\title{
High Performance of a Chiral Diene-Rhodium Catalyst for the Rhodium-Catalyzed Asymmetric 1,4-Addition of Arylboroxines
}

\author{
Fu-Xue Chen, Asato Kina, and Tamio Hayashi* \\ Department of Chemistry, Graduate School of Science, Kyoto University, Sakyo, \\ Kyoto 606-8502, Japan
}

\section{Experimental}

\section{General}

All anaerobic and moisture-sensitive manipulations were carried out with standard Schlenk techniques under predried nitrogen or glovebox techniques under prepurified argon. NMR spectra were recorded at $500 \mathrm{MHz}$ for ${ }^{1} \mathrm{H}$ and $125 \mathrm{MHz}$ for ${ }^{13} \mathrm{C} \mathrm{NMR}$. Chemical shifts are reported in $\delta$ ppm referenced to an internal $\mathrm{SiMe}_{4}$ standard or the residual peak of THF $(\delta 3.76)$ for ${ }^{1} \mathrm{H}$ NMR and chloroform- $d(\delta 77.05)$ or THF- $d_{8}(\delta 67.0)$ for ${ }^{13} \mathrm{C}$ NMR. Optical rotations were measured on a JASCO DIP-370 polarimeter. $\mathrm{CHCl}_{3}$ was distilled over $\mathrm{P}_{2} \mathrm{O}_{5}$ under $\mathrm{N}_{2}$. THF and 1,4-dioxane were distilled over benzophenone-ketyl under $\mathrm{N}_{2} . \mathrm{H}_{2} \mathrm{O}$ was degassed by bubbling of $\mathrm{N}_{2}$.

2-Cyclohexenone and 2-cyclopentenone were distilled before use. Phenylboronic acid (Tokyo Kasei Kogyo Co., Ltd.) was used as received. Arylboroxines were prepared from purchased arylboronic acids (vide infra). $\left[\mathrm{RhCl}\left(\mathrm{C}_{2} \mathrm{H}_{4}\right)_{2}\right]_{2}{ }^{1}$ and $(S, S)-\mathrm{Bn}$-bod*2 were prepared following the reported procedures.

\section{Preparation of $\left[\operatorname{RhCl}\left((S, S)-\mathrm{Bn}-\operatorname{bod}^{*}\right)\right]_{2}(1)$}

A suspension of $\left[\mathrm{RhCl}\left(\mathrm{C}_{2} \mathrm{H}_{4}\right)_{2}\right]_{2}(43 \mathrm{mg}, 0.22 \mathrm{mmol})$ and $(S, S)$-Bn-bod* $(60 \mathrm{mg}, 0.21$ mmol $)$ in $\mathrm{CHCl}_{3}(10 \mathrm{~mL})$ was stirred for $2 \mathrm{~h}$ at room temperature. The mixture was filtered through celite pad. The solvent of the filtrate was removed under reduced pressure to give $89 \mathrm{mg}$ of rhodium complex in a quantitative yield as a yellow solid.

${ }^{1} \mathrm{H} \mathrm{NMR}\left(\mathrm{CDCl}_{3}\right): \delta 0.24(\mathrm{~m}, 4 \mathrm{H}), 0.52(\mathrm{~m}, 4 \mathrm{H}), 2.76(\mathrm{~d}, J=14.1 \mathrm{~Hz}, 4 \mathrm{H}), 3.52(\mathrm{br}, 4 \mathrm{H}), 3.54$ $(\mathrm{d}, J=14.0 \mathrm{~Hz}, 4 \mathrm{H}), 4.03(\mathrm{~d}, J=5.4 \mathrm{~Hz}, 4 \mathrm{H}), 7.21(\mathrm{t}, J=7.3 \mathrm{~Hz}, 4 \mathrm{H}), 7.29$ (t, $J=7.4 \mathrm{~Hz}, 8 \mathrm{H})$, $7.35(\mathrm{~d}, J=7.4 \mathrm{~Hz}, 8 \mathrm{H}) ;{ }^{13} \mathrm{C} \mathrm{NMR}\left(\mathrm{CDCl}_{3}\right): \delta 25.9,43.1,45.6,50.2\left(\mathrm{~d}, J_{\mathrm{C}-\mathrm{Rh}}=10.9 \mathrm{~Hz}\right), 69.6$ 
$\left(\mathrm{d}, J_{\mathrm{C}-\mathrm{Rh}}=11.4 \mathrm{~Hz}\right), 126.4,128.4,128.9,138.8$; Anal. Calcd for $\mathrm{C}_{44} \mathrm{H}_{44} \mathrm{Cl}_{2} \mathrm{Rh}_{2}: \mathrm{C}, 62.21, \mathrm{H}$, 5.22: found $\mathrm{C}, 61.93 ; \mathrm{H}, 5.32 ;[\alpha]^{\mathrm{D}_{25}}+571\left(c 0.769, \mathrm{CHCl}_{3}\right)$.

\section{Preparation of phenylboroxine $(5 \mathrm{~m})$}

A solution of phenylboronic acid $(5.0 \mathrm{~g}, 40 \mathrm{mmol})$ in benzene $(100 \mathrm{~mL})$ was refluxed for 2 h, during which $\mathrm{H}_{2} \mathrm{O}$ was removed azeotropically. The mixture was concentrated under reduced pressure into ca. $30 \mathrm{~mL}$, and it was cooled to room temperature. The precipitate obtained by filtration was washed 5 times with hexane to give $3.0 \mathrm{~g}$ of boroxine $\mathbf{5 m}$ ( $71 \%$ yield) as a white crystal.

${ }^{1} \mathrm{H} \mathrm{NMR}\left(\mathrm{CDCl}_{3}\right): \delta 7.50(\mathrm{~m}, 6 \mathrm{H}), 7.60(\mathrm{~m}, 3 \mathrm{H}), 8.25(\mathrm{dd}, J=8.0 \mathrm{~Hz}, 1.3 \mathrm{~Hz}, 6 \mathrm{H})$.

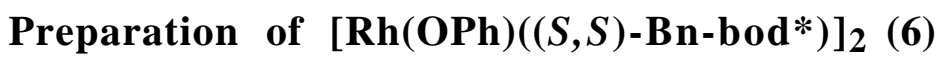

The procedure reported for $[\mathrm{Rh}(\mathrm{OPh})(\mathrm{cod})]_{2}{ }^{3}$ was modified as follows. A solution of $\left[\operatorname{RhCl}\left((S, S)-B n-\text { bod }^{*}\right)\right]_{2}(10.3 \mathrm{mg}, 24.3 \mu \mathrm{mol})$ and potassium phenoxide $(3.21 \mathrm{mg}, 24.3 \mu \mathrm{mol})$, which was prepared from phenol and potassium hydride, in THF- $d_{8}(0.5 \mathrm{~mL})$ was heated at $40{ }^{\circ} \mathrm{C}$ for $1 \mathrm{~h}$. The formation of phenoxide-dimer 6 was determined by ${ }^{1} \mathrm{H}$ and ${ }^{13} \mathrm{C}$ NMR. The solvent was removed under reduced pressure. To the residue was added $\mathrm{CH}_{2} \mathrm{Cl}_{2}$ and it was filtered through celite for removal of the salt to give a quantitative yield of phenoxide-dimer $\mathbf{6}$, which is contaminated with a small amount of impurity (less than ca. $5 \%$ by ${ }^{1} \mathrm{H}$ NMR). The crude sample was used for the next reaction as a catalyst without further purification.

${ }^{1} \mathrm{H}$ NMR (THF- $\left.d_{8}\right): \delta 0.26(\mathrm{~m}, 4 \mathrm{H}), 0.37(\mathrm{~m}, 4 \mathrm{H}), 1.76(\mathrm{~d}, J=13.8 \mathrm{~Hz}, 4 \mathrm{H}), 2.78(\mathrm{~d}, J=6.1$ $\mathrm{Hz}, 4 \mathrm{H}), 2.98(\mathrm{~d}, J=14.0 \mathrm{~Hz}, 4 \mathrm{H}), 3.90(\mathrm{~d}, J=5.6 \mathrm{~Hz}, 4 \mathrm{H}), 6.72(\mathrm{t}, J=7.2 \mathrm{~Hz}, 2 \mathrm{H}), 7.09(\mathrm{~d}, J$ $=7.6 \mathrm{~Hz}, 4 \mathrm{H}), 7.15(\mathrm{t}, J=7.6 \mathrm{~Hz}, 4 \mathrm{H}), 7.31(\mathrm{t}, J=7.4 \mathrm{~Hz}, 4 \mathrm{H}), 7.41(\mathrm{t}, J=7.6 \mathrm{~Hz}, 8 \mathrm{H}), 7.46$ $(\mathrm{d}, J=7.8 \mathrm{~Hz}, 8 \mathrm{H}) ;{ }^{13} \mathrm{C}$ NMR $\left(\mathrm{THF}-d_{8}\right): \delta 25.2,39.7,44.2,44.7\left(\mathrm{~d}, J_{\mathrm{C}-\mathrm{Rh}}=11.4 \mathrm{~Hz}\right), 61.9(\mathrm{~d}$, $\left.J_{\mathrm{C}-\mathrm{Rh}}=11.9 \mathrm{~Hz}\right), 115.2,119.0,122.2,125.8,128.0,128.9,139.5,166.0 ;[\alpha]^{\mathrm{D}_{25}}+945^{\circ}(c$ $0.665, \mathrm{THF})$.

\section{Catalytic asymmetric 1,4 -addition of arylboron reagents to $\alpha, \beta$-unsaturated}

\section{ketones}


As a typical procedure, the addition of phenylboroxine (5m) to 2-cyclohexenone (2a) (entry 8 in Table 1) is shown below: To a suspension of $\left[\mathrm{RhCl}\left((S, S)-\mathrm{Bn}-\text { bod }^{*}\right)\right]_{2}(0.13 \mathrm{mg}, 0.30 \mu \mathrm{mol}$ $\mathrm{Rh})$ and phenylboroxine $(\mathbf{5 m}, 371 \mathrm{mg}, 3.60 \mathrm{mmol} \mathrm{B})$ in $3.0 \mathrm{~mL}$ of 1,4-dioxane was added 2cyclohexenone (2a, $288 \mathrm{mg}, 3.00 \mathrm{mmol})$ and aqueous $\mathrm{KOH}(5.0 \mathrm{M}, 0.30 \mathrm{~mL}, 1.5 \mathrm{mmol})$. The solution was stirred at $30{ }^{\circ} \mathrm{C}$ for $1 \mathrm{~h}$. The resulting mixture was passed through a short silica gel pad and eluted with $\mathrm{Et}_{2} \mathrm{O}$. Evaporation of the solvent followed by preparative TLC (silica gel, hexane/ethyl acetate $=3 / 1)$ gave $(S)$-3-phenylcyclohexanone (4am) $(501 \mathrm{mg}, 96 \%$ yield, $96 \%$ ee $)$ as a colorless oil. All of 1,4-addition products were reported in the literature. ${ }^{4}$

(1) Cramer, R. Inorg. Synth. 1974, 15, 14.

(2) Otomaru, Y.; Okamoto, K.; Shintani, R.; Hayashi, T. J. Org. Chem. 2005, 70, 2503.

(3) Kostas, I. D.; Vallianatou, K. A.; Kyritsis, P.; Zedník, J.; Vohlídal, J. Inorg. Chem. Acta 2004, 357, 3084.

(4) Takaya, Y.; Ogasawara, M.; Hayashi, T.; Sakai, M.; Miyaura, N. J. Am. Chem. Soc. 1998, 120,5579 . 

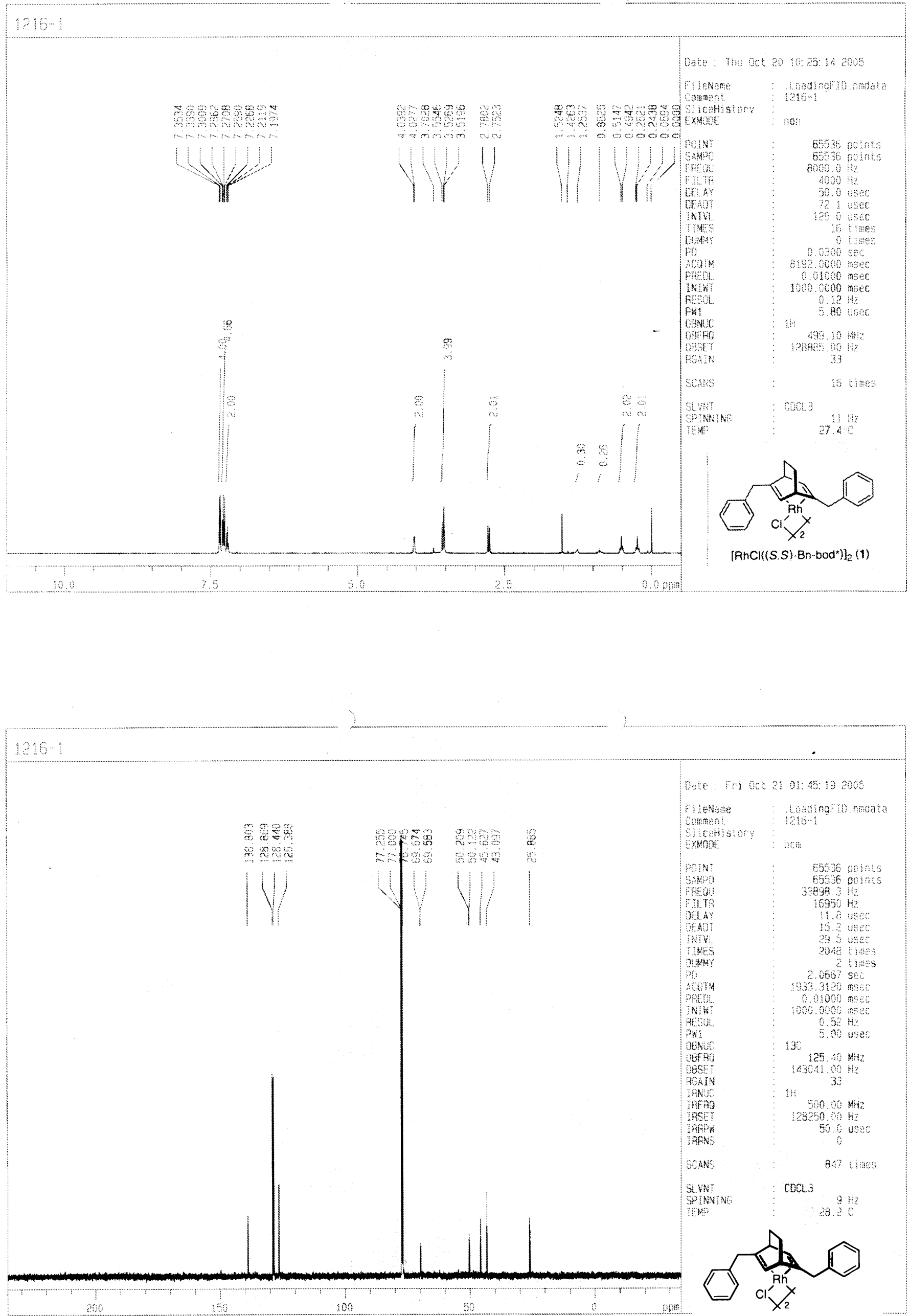

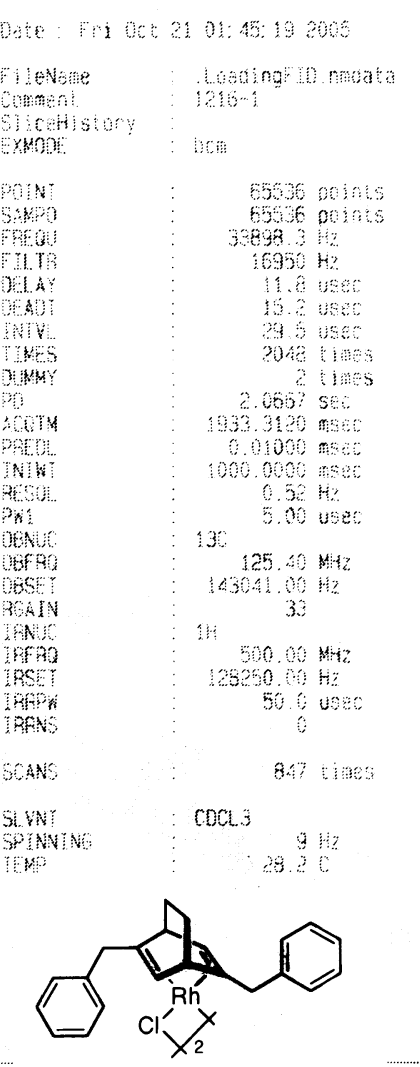

$\left[\mathrm{RhCl}\left((S, S)-\mathrm{Bn}-\text { bod }^{\star}\right)\right]_{2}(1)$ 

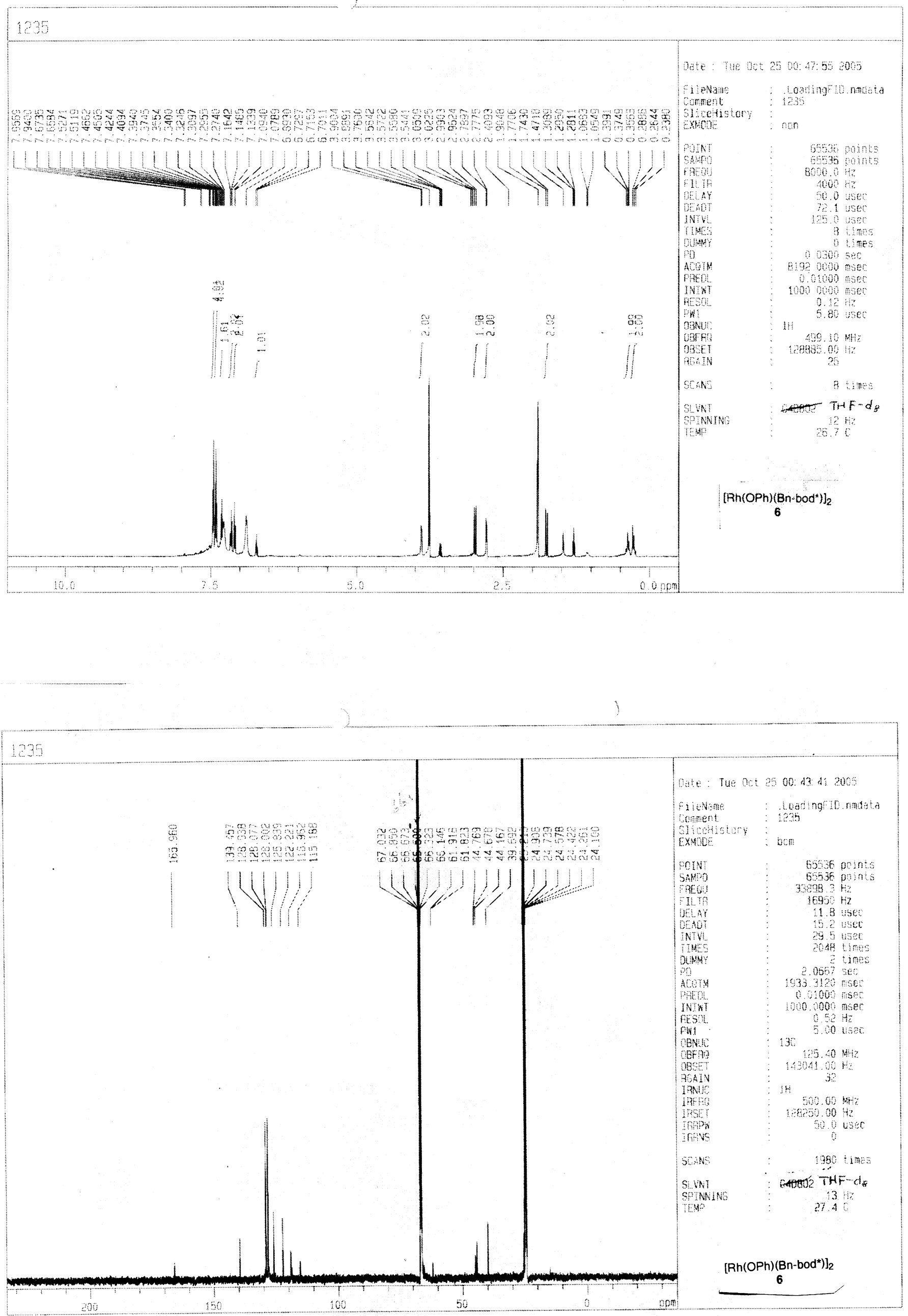